\title{
Design synthesis of complex ship structures
}

\section{Vedran Zanic , Jerolim Andric \& Pero Prebeg}

To cite this article: Vedran Zanic , Jerolim Andric \& Pero Prebeg (2013) Design synthesis of complex ship structures, Ships and Offshore Structures, 8:3-4, 383-403, DOI: 10.1080/17445302.2013.783455

To link to this article: http://dx.doi.org/10.1080/17445302.2013.783455

\section{曲 Published online: 23 May 2013.}

Submit your article to this journal ๘

\section{山 Article views: 111}

Q View related articles $\square$

Citing articles: 3 View citing articles $\square$ 


\title{
Design synthesis of complex ship structures
}

\author{
Vedran Zanic, Jerolim Andric and Pero Prebeg* \\ Faculty of Mechanical Engineering and Naval Architecture, University of Zagreb, Zagreb, Croatia
}

(Received 4 December 2012; final version received 28 February 2013)

\begin{abstract}
The paper presents the principal steps in the definition of a practical design model for multi-criteria synthesis of complex thin-walled ship structures in concept and preliminary design. It elaborates the general requirements on the design procedure, balanced and applicable combinations of design models and a practical example of the basic analysis models/IT modules within the MAESTRO/OCTOPUS design support system. System identification from the multi-stakeholder perspectives of owner and society, and the formulation and solution to the dimensionally large-scale structural designs are presented as a step towards the practical implementation of multi-criteria decision-making in ship design practice since only a joint effort could lead to the satisfaction of all stakeholders in today's challenging ship-building industry. The practical structural design procedure/methodology, capable of embedding multiple design quality criteria, is demonstrated using a benchmark case study on the innovative RoPax ship.
\end{abstract}

Keywords: design support system (DeSS); Pareto supported decision-making (PSD); thin-walled ship structures; structural design procedure; structural optimisation; RoPax design

\section{Introduction}

The design of complex ship thin-walled structures falls within the category of large-scale problems, characterised by several design objectives, hundreds of design variables and a large number of design constraints, interwoven with the full-ship 3D structural (e.g. FEM) and load (e.g. stochastic formulation) models. In practice, the dimensionality may be of order: (a) number of design variables: $\approx 5-600$, (b) number of criteria functions: $\approx\left[n^{\text {Panelrows }}(\approx 500) \times n^{\text {DesignLC }}\right.$ $\left.(\approx 20) \times n^{\text {FailureCriteria/Panel in a row }}(\approx 20)\right]$ and (c) driven with 5-6 design objectives.

The first objective of the paper is to present techniques applicable for the practical design of complex ship structures in the acceptable time.

The second focus of the paper is the presentation of the efficient design procedure capable of solving the design problems (DPs) with multiple objectives of a real ship structure in cooperation with the owner, yard and Classification Society.

Mathematical DP should be formulated together with the associated solvable mathematical model and corresponding IT modules to support multiple stakeholders in their design-related decision-making.

Such models, when implemented, may be called design support systems (DeSS), combining the efficient design analysis model for design response calculation and evaluation and the synthesis model for the design-related subjective and objective decision-making. Integration of available techniques into the interactive computing envi- ronment, for the multi-criteria design, is presented as an additional step towards the effective and efficient interaction between stakeholders and DP, within the given design time frame.

Pareto hyper-surface of non-dominated designs can be of a great assistance and can support the design-related decision-making by presenting stakeholders with a small number of Pareto optimal designs (Yu 1985) that may be subjected to their revealed (or possible) preferences in the selection of the preferred design, and not the infinity of possible feasible solutions.

In order to enable practical application of such DeSS and due to the complexity of the problem, it is necessary to include simplifications/manipulations of the original problem, multi-fidelity analysis tools, problem decomposition with a coordinated solution of decomposed subproblems and an application of customised multicriteria decision-making techniques, as presented in the paper.

The second section is devoted to the practical identification and formulation of the ship structural DP. In addition to the consideration of selection of design variables and design criteria functions, the section elaborates the importance of a loading model and the handling of a ship structural analysis model from a design point of view.

The third section deals with the application of multicriteria decision-making techniques, with a special consideration for the multi-objective optimisation methods capable of generating the Pareto frontier.

${ }^{*}$ Corresponding author. Email: pero.prebeg@fsb.hr 
The fourth section elaborates the DP formulation for complex structures with a large number of design variables and design criteria. Problem manipulations towards efficient solution strategies, surrogate modelling and problem decomposition with coordinated solution of subproblems are presented as a way to improve solution quality.

Integration of these techniques into the interactive computing environment, for the multi-criteria design, is presented as an additional step towards the effective interaction between the designer and the DP within the given design time frame.

The fifth section is devoted to the efficient solution of the DP where the applicable design procedures are presented for the concept design phase (denoted CDP) and preliminary design phase (denoted PDP), using the practical example of a RoPax ship. Each design step includes a number of analysis and synthesis modules, creating the practical environment supporting the designer's decision-making tasks. Techniques of objective (based on optimisation utilities) and subjective decision-making (on the generated Pareto frontier) are applied.

Sensitivity analysis of the influence of safety taken as one of the objectives (not only as a constraint) on structural design shows that, for the same owner's gain, it is possible to increase design safety by means of the appropriate procedure, and satisfy both the owner's and societal constraints as formulated in references ISSC (2009) and Zanic, Kitarovic, et al. (2010) and summarised in the Editorial of this special issue of the Journal (Zanic 2013).

Taxonomy used in this paper is developed for the DP formulation (Zanic, Andric, et al. 2010) and extended in the Editorial.

\section{DP identification and formulation}

DP definition includes (a) DP identification on the conceptual level, (b) design model formulation on the algorithmic level and (c) DP solution on the procedural level.

Identification of the practical DP implies the selection of:

- variables (as subsets of descriptors) coming from, e.g., computer-assisted design (CAD) models or initial drawings;

- criteria functions (constraints and attributes) coming from, e.g., Classification Society Rules or yard;

- objectives (attributes with direction of improvement) from the owner and society, all defined on the respective mathematical support structure, as presented next.

\subsection{Design descriptors/variables, control structures, generic models}

In the structural design subproblem of the ship design process, the analysis models (AMs) are structural 'solvers', within the design synthesis procedure, and they require model description (sets of descriptors) for each design variant.

AMs for the calculation of criteria function values are implemented into response $(\rho)$, feasibility $(\alpha, \pi)$, and quality $(\Omega)$ design criteria-based IT modules. Such ITimplemented mathematical models are denoted in this paper as modules. They are briefly presented in Table 1 , containing available modules, for two practical ship structural design systems (OCTOPUS and MAESTRO):

- OCTOPUS (OCTOPUS 2012) is an integrated ship structural modelling, analysis and decision support system for the CDP. OCTOPUS-Analyzer model (2.5D finite-element model [FEM]) is generated on the basis of one-bay model produced using the MAESTRO Modeler software and/or by automated CAD to FEM data transfer. It can be used for fast concept exploration, on the midship section level in $\mathrm{CDP}$, and is a rapid first step to the final determination of the structural scantlings using MAESTRO software in the PDP. OCTOPUS-Designer, as a tool for decision-making (providing interactive design environment with different optimisers, sequencers etc.) has been applied in both design phases.

- MAESTRO (MAESTRO 2012) is an integrated ship structural modelling, analysis and optimisation system for the PDP. It combines rapid ship-oriented structural modelling, large-scale global- and finemesh 3D FE analysis, structural failure evaluation and structural optimisation. MAESTRO's core capabilities represent a system for rationally based optimum design of large, complex thin-walled structures.

Practical formulation of the AM, for the given loads, includes only two sets of entities, design descriptors/properties and design criteria (design functions/ mappings), that have to be identified as the basic DP components in the DeSS.

Descriptors $\mathbf{d}$ are the arguments of the criteria functions, formulated within the response, adequacy and quality models.

Control structure is a minimal part of ship structure, sufficient for DP formulation, represented by the subset of design descriptors $\mathbf{d}^{C S} \subseteq \mathbf{d}$.

To capture, accurately enough, the critical values of the design criteria (objectives and constraints using $\Omega$ and $\alpha$ models), based upon structural response fields generated by the $\rho$-models, the different 'control structures' were used by profession. Typical examples are:

- 2 D midship section models required by the Classification Society Rules for certain ship types. It has been established that the responses of modern complex ships can only be accurately calculated by applying 
Table 1. List of the available modules of the analysis model in MAESTRO and OCTOPUS structural design software.

List of the available modules of the analysis model in MAESTRO and OCTOPUS software

\begin{tabular}{|c|c|c|}
\hline Meta-system & $\begin{array}{l}\text { MAESTRO (M) and OCTOPUS Analyzer (O) } \\
\text { modules (implemented mappings) }\end{array}$ & $\begin{array}{c}\text { Description of the MAESTRO (M) and OCTOPUS (O) } \\
\text { analysis math. models/mappings }\end{array}$ \\
\hline $\begin{array}{l}\text { Physical } \\
(\Phi)\end{array}$ & $\begin{array}{l}\text { M: MAESTROFEM structural modeler } \\
\text { O: MAESTRO FEM structural modeler }\end{array}$ & $\begin{array}{l}\text { MAESTRO Modeler is used to define generic 3D } \\
\text { (MAESTRO) FEM models; also used for 2.5D } \\
\text { (OCTOPUS) for specified cross-sections (web-frame, } \\
\text { bulkhead) }\end{array}$ \\
\hline $\begin{array}{l}\text { Environment } \\
\qquad(\varepsilon-1, \ldots, \mathrm{LC})\end{array}$ & $\begin{array}{l}\text { M: MAESTRO 3D FEM loader } \\
\text { O: OCTLOAD - Classification Society Rules } \\
\text { (IACS) loads module }\end{array}$ & $\begin{array}{l}\text { Classification Society loads or loads sea-keeping } \\
\text { analysis. 3D load distributions are automatically } \\
\text { generated. }\end{array}$ \\
\hline $\begin{array}{l}\text { Response } \\
\qquad(\rho 3 \mathrm{D})(\rho-1)\end{array}$ & $\begin{array}{l}\text { M: MAESTRO 3D FEM solver } \\
\text { O: LTOR - calculates primary strength fields } \\
\quad \text { (warping displacements, normal/shear stresses) }\end{array}$ & $\begin{array}{l}\text { Full 3D FEM solver using FE and macroelements. } \\
\text { Extended beam theory (cross-section warping fields via } \\
\text { FEM in vertical/horizontal bending and warping } \\
\text { torsion) }\end{array}$ \\
\hline $\begin{array}{l}\text { Response } \\
\qquad(\rho-2,3)\end{array}$ & $\begin{array}{l}\text { O: TOKV \& TBHD - secondary strength fields: } \\
\text { transverse and lateral displacements; stresses }\end{array}$ & $\begin{array}{l}\text { FEM analysis of web-frame and bulkhead using beam } \\
\text { element with rigid ends and stiffened shell } \\
\text { isoparametric eight-node macroelement. }\end{array}$ \\
\hline \multirow[t]{2}{*}{$\begin{array}{l}\text { Adequacy/feasibility } \\
\qquad(\alpha-1,3)\end{array}$} & $\begin{array}{l}\text { M: ULSAP, EVAL - libraries of analytical safety } \\
\text { criteria }\end{array}$ & $\begin{array}{l}\text { Calculation of 'patch' feasibility based on (M: } \rho 3 \mathrm{D} \\
\text { responses fields sorted into libraries of analytical } \\
\text { safety criteria; Hughes and Paik (2010)) }\end{array}$ \\
\hline & $\begin{array}{l}\text { O: EPAN library of stiffened panel and girder } \\
\text { ultimate strength and serviceability criteria and } \\
\text { rules, fatigue calculation }\end{array}$ & $\begin{array}{l}\text { Calculation of macroelement feasibility based on } \\
\text { super-position of response fields (O: } \rho-1, \rho-2, \rho- \\
\text { 3) sorted into libraries of analytical safety criteria } \\
\text { and rule-defined criteria (CSR IACS) }\end{array}$ \\
\hline \multirow[t]{3}{*}{$\begin{array}{l}\text { Adequacy } \\
\qquad(\alpha-3,4,5)\end{array}$} & M: ALPS/HULL - ultimate longitudinal strength & $\begin{array}{l}\text { Paik and Thayamballi (2003)-based procedure inbuilt } \\
\text { into MAESTRO. }\end{array}$ \\
\hline & O: LUSA - ultimate longitudinal strength & $\begin{array}{l}\text { Incremental ultimate strength analysis using the } \\
\text { extended Smith procedure. }\end{array}$ \\
\hline & $\begin{array}{l}\text { O: MIND - Classification Society Rules minimal } \\
\text { dimensions criteria }\end{array}$ & $\begin{array}{l}\text { Minimal dimensions definition from Classification } \\
\text { Society Rules (CSR IACS) }\end{array}$ \\
\hline \multirow[t]{3}{*}{$\begin{array}{l}\text { Reliability } \\
\qquad(\pi-1,2,3)\end{array}$} & $\begin{array}{l}\text { O: US-3 reliability calculation of element and } \\
\text { system failure probability (levels } 1-3 \text {, } \\
\text { mechanism) }\end{array}$ & $\begin{array}{l}\text { FORM approach to panel reliability. } \beta \text {-unzipping } \\
\text { method used to determine system probability of } \\
\text { failure. }\end{array}$ \\
\hline & O: SENC sensitivity to correlation of input variate & Sensitivity calculation based on Nataf model \\
\hline & $\begin{array}{l}\text { O: RELUS - ultimate strength reliability } \\
\text { calculation }\end{array}$ & $\begin{array}{l}\text { Fast reliability assessment by dimension reduction } \\
\text { approach }\end{array}$ \\
\hline \multirow[t]{6}{*}{$\begin{array}{l}\text { Quality KPI } \\
\qquad(\Omega-1, \ldots, 9)\end{array}$} & (1-2) M \& O:WGT/CST - weight/cost modules & $\begin{array}{l}\text { Minimal structural weight }=\text { max. DWT increase; min. } \\
\text { initial cost }\end{array}$ \\
\hline & $\begin{array}{l}\text { (3) O: MUH / MUS - ultimate hull girder bending } \\
\text { moment }\end{array}$ & Calculations using LUSA and RELUS \\
\hline & (4) O: URL - ultimate racking load & Deterministic calculation using US-3 and TOKV \\
\hline & (5) O: FLIFE - fatigue life & $\begin{array}{l}\text { Fatigue life calculation for longitudinal-web frame } \\
\text { intersection }\end{array}$ \\
\hline & (6) O: UDBP/(7) UDBR - reliability measures & $\begin{array}{l}\text { (Upper Ditlevsen bound: panel or racking failure } \\
\text { problems in testing) }\end{array}$ \\
\hline & (8) $\mathrm{O}: \mathrm{GML} /(9) \mathrm{TSN}$ - robustness measures & Information context measure/Taguchi SNR via FFE \\
\hline
\end{tabular}

the 3D FE full ship models. Equivalent 1D (ship as a beam) or $2 \mathrm{D}$ models are not only too restricted but also require additional 'idealisation time', so that their somewhat greater simplicity is lost in the additional work and pending inaccuracy problems.

- The standard partial 3-hold FE models (with one FE between stiffeners) used for prismatic structures, see current IACS Rules for Tankers and/or Bulk Carriers, have the modelling precision on the PDP level. They are unnecessarily detailed (too large descriptor sets) and therefore time-consuming regard- ing generation/computation for the design concept exploration.

The primary objective is to provide structural designers in the CDP with a practical approach that is capable of fast modelling and analysis of complex structural responses, including 3D effects, for different topological concepts generated in multi-criteria optimisation process.

- Generic ship structural model has been introduced for the application in DP formulation for the CDP, as a possible solution to those requirements (Andric 


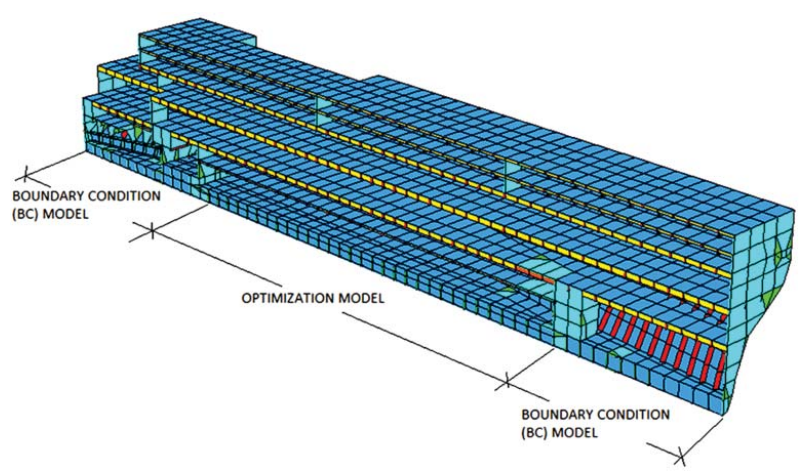

Figure 1. Typical basic generic 3D FE model for CDP. (This figure is available in colour online.)

and Zanic 2010). To generate the complex responses as fast as possible, the generic ship model geometry has to follow the cross-section characteristics and the general layout. An extruded/tapered box model with transverse/longitudinal bulkheads is enabling fast development of generic 3D FE models. They are based on plated building blocks (mainly stiffened panels) and the equivalent pre-modelled parts for large side openings. This requires accurate modelling focused only on the 'controlling' part of the structure. The structural elements' boundary supports (e.g. brackets, local supports etc.) must be included for the correct definition of maximal element capacity over local element length. Generic model length should extend approximately over the full length and depth of the ship to correctly model, e.g., superstructure deck effectiveness.

To assure accurate results in the design model, usually three submodels have to be generated (see Figure 1), i.e.:

- design model or 'control' structure obtained by extrusion of the characteristic cross-section and

- two models used for the adjustment of boundary conditions (BCs) at the ends of the design model.

Topological level modifications (such as the side openings, the number longitudinal bulkhead, the topology of superstructure, deckhouse, recess etc.) are easily implemented using the generic model. The selection of appropriate topological concepts and structural scantlings is necessary for the approximate assessment of the structural weight, the centre of gravity $(\mathrm{CG})$ and the achievable clearances (with respect to height of deck beams and girders etc.). The basic goal is to generate simultaneously the acceptable structural layout and feasible structural design; see Zanic et al. (2009).

Geometrical changes on the extruded FEM model are very fast since they require only very limited data changes (e.g. value of spacing between decks, size of size openings, geometry of superstructure, deckhouse, recess etc.).

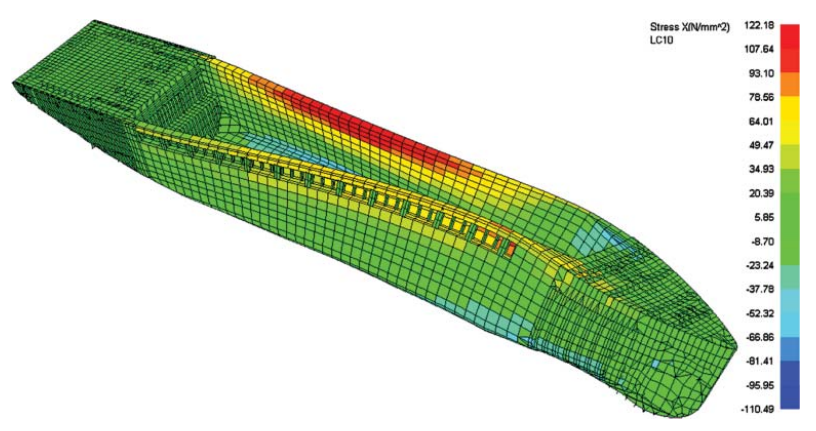

Figure 2. CDP/PDP model for deflection control between two $\mathrm{BC}$ models of peaks. (This figure is available in colour online.)

$B C$ models mainly include ship segments (aft and fore peaks, engine room etc.) that have to be fully defined in the later stages of the design process. Those segments can be modelled approximately, by the crude modifications of the extruded model, to provide the adequate or the preferred $\mathrm{BCs}$ at the ends of the 'control' structure. They can also be obtained from existing similar ships and statically condensed as super-elements. Sometimes, they can be obtained by automatic CAD-to-FEM transfer, e.g., NAPASteel-to-MAESTRO developments; see Holmberg and Hunter (2011). Figure 2 presents such BC models generated and used to provide realistic $\mathrm{BCs}$ for the calculation of deflection constraints imposed, e.g., by the hatch-covers manufacturers.

Design descriptors/properties can be understood as an entire input data for the design software regarding the object to be designed (minimal model description). It consists of: design descriptors/properties such as topology, geometry, material and scantlings, represented by the appropriate sets of the concrete design.

Most modern design procedures include CADsupported product definition and FEM-based analysis/evaluation. Transformation between CAD and FEM models may be difficult, although the two models are adjunct. In this context, descriptor(s) defined in the parametric CAD model will influence only a subset of elements in the FEM response model.

Properties or descriptors of a particular design will therefore instantiate the group of their supporting entities (FEM elements) that have the same property value in the specific part of the structure. Such groups, belonging to the specified property (descriptor $d_{i}$ or set $\mathbf{d}^{\mathrm{k}}$ ) value may be denoted in general as descriptor/property element group (denoted $d e G$ or $p e G$ ).

In a similar manner, the groups of elements acting as a support for certain design criterion function/mapping may be denoted as criterion-based element groups (ceG), e.g., group of elements participating in weight calculation, group of elements supporting ultimate strength calculation, patches of elements supporting failure modes in the local structure among strong girders/boundaries and 
criteria supporting reliability and risk calculation (ship, substructures).

Note that in the first version of MAESTRO (Hughes et al. 1980), the concept of strake was introduced by O.F. Hughes, where peGs and ceGs for properties, objectives and constraints conveniently coincided.

Index space, see LS INGRID (1998), enables a very comprehensive design description; see Andric and Zanic (2010). It is a compact structural description particularly related to the support of different descriptors and criteria on the ship's 3D grid (spacing) level by, e.g., frame number, deck number etc. It is spanned upon the ship girder systems and spatial distributions of strong substructures (ship envelope, decks, T/L bulkheads etc.). Principles of $1 \mathrm{D}, 2 \mathrm{D}$ and $3 \mathrm{D}$ extrusion can be conveniently applied by extruding the 1D line of the longitudinal spacing upwards, defining the cross-section 2D grid, and extruding it along the ship's length to generate a prismatic or tapered 3D model.

The modelling can be simplified using building blocks that are repetitive and replaceable. The minimal replaceable building blocks for the CDP level are stiffened panels (span could reach, e.g., deck to deck in ship sides) and complex bracketed beams forming together plated building blocks. Modification of the structure is then performed by the controlled addition or deletion of sets of building blocks in the index space (e.g. recesses, side plating, atriums etc.). Those building blocks (FE, macroelements) should have an adequate degree of FEM shape functions to accurately generate the deformation and failure modes while minimising their number.

Design of experiments (DOE) techniques (FFE, orthogonal arrays, analysis of variance [ANOVA] etc.); see Montgomery (2001), could be easily used, on generic model level, to systematically study multiple topological parameters simultaneously; see Ross (1988). It enables a rational identification of the dominant parameters on the topological level and provides the designer with the preliminary value of each topological parameter; see Andric (2007). They give a better starting point for further determination of scantlings based on the DeSS.

Finally, generic models are suitable for further development by means of updating and re-meshing in the final stage of the concept optimisation and evaluation process. The qualitative and quantitative decisions can be made on the basis of its results before the full-ship FE model is fully developed and evaluated.

\subsection{Identification of criteria functions and design loads}

After design descriptors are defined on the control structure, it is necessary to identify design criteria functions set and complete the DP identification (taxonomy is given in the Editorial of this special issue).
Design criteria (quality measures) $\mathbf{q}=\left\{q_{i}\right\}$ are defined using the set (library) of design criteria functions/mappings $\mathbf{c}=\left\{\mathrm{c}_{i}().\right\}$, e.g., for structural cost, weight, safety, evaluations etc. Achieved values of design qualities are obtained accordingly as $\mathbf{q}=\{\mathbf{c}(\mathbf{d})\}$. The criteria functions/mappings will be discussed and applied in the examples in the following sections. Criteria functions/mappings are subdivided into subsets of:

- constraint functions $g_{j}($.$) for definition of the crisp de-$ sign constraints $g_{j}() \geq$.0 based on the direct calculation criteria for global and local strength through analytical formulas and/or Classification Society Rules (e.g. safety constraints). Satisfaction of constraints may also be defined with adequacy indicator $I_{g}$ (pass, fail);

- design attribute functions $\mathrm{a}_{j}($.$) for definition of design$ attributes (e.g. costs, weight, safety measures) that can be manipulated into design objective functions $\mathrm{o}_{j}()=.\mathrm{f}\left(\mathrm{a}_{j}().\right)$ when direction of quality improvement is defined and when non-discriminatory parts are eliminated (e.g. administrative costs that are the same for all design variants);

- subjective criteria functions $\mathrm{u}_{j}($.$) or \mathrm{v}_{j}($.$) for final de-$ sign selection. The latter two are formed by interactively introducing subjectivity and uncertainty that characterise decision-making of the realistic design process.

Design loads selection is one of the most important parts of design procedure since 'optimistic' loads would lead to unsafe structure, while 'pessimistic' ones would lead to oversized design.

Design loads (extreme and fatigue) in the CDP of structural design are usually defined by the Classification Society Rules. In the case of direct calculation of loads, they are usually defined in the general design CDP where ship form and dimensions are determined, what usually precedes structural CDP.

For the realistic definition of the 'worst' combination of load components, the concept of 'design pressure' is a very convenient one. In general, given the Classification Society Rules loading data descriptors $\mathbf{d}^{\text {C.RULES }}$, loads should be transformed into a model format based on appropriate mapping and applied to the respective $\rho$ and $\alpha$ model.

\section{Multi-criteria decision-making and solution strategies}

Multi-criteria decision-making (MCDM) is widely accepted as the realistic engineering approach in the presence of multiple, usually conflicting objectives. It can be defined as the body of methods and procedures by which multiple conflicting criteria can be formally incorporated into the rational design process. 
Selection of MADM (multi-attribute decision-making) or MODM (multi-objective decision-making) formulation depends on many aspects of the DP such as dimensionality, mathematical complexity, availability of specific tools etc.

DeSS, as explained in the Editorial, seems to be a reasonable approach for this type of problems. In order to enable practical implementation of Pareto supported decision-making (PSD) or decision support problem (DSP) approach, it is necessary to introduce insight into the techniques and procedures available. Due to the complexity of the problem, it is necessary to develop additional techniques in order to apply DeSS in a tight and rigid time frame that is usually imposed on the designers.

\subsection{MODM approach}

The techniques for the highly non-linear and highdimensional problems necessarily lead to a variety of methods in operations research closely tailored to the characteristics of objective and constraint functions of the problem at hand. Design mapping in MODM is usually transformed into the standard mathematical programming formulation:

$$
\operatorname{Max} v(\mathbf{x}) \text { such that } \mathbf{x} \in \mathbf{X}^{\geq} \text {. }
$$

If a value function $v(-)$, combining multiple objectives, can be constructed, the methods for single compound objective could be used, e.g., compromise and goal programming methods.

Dualisation (see Subsection 4.1) is one of the most frequently used manipulations in practical MODM applications, especially in structural design applications:

- Rigo and Fleury (2001) and Rigo (2001) avoid the main difficulty in solving a dual problem i.e., dealing with non-linear and implicit constraints. To avoid a large number of time-consuming re-assessments of these non-linear and implicit functions, they suggest applying convex approximations.

- MAESTRO (2012) uses dual formulation of sequential linear programming (Hughes et al. 1980), with accumulation of linearised constraints for non-linear feasibility criteria and special linearisation technique (including second-order terms) in generating failure hyper-planes.

Basic MODM strategies used to solve manipulated problems are:

- Iterative and piece-wise strategy (leading to a sequence of simple problems, e.g., feasible directions, penalty function approach etc.).

- Relaxation strategy (temporarily removing some constraints, e.g., in dualisation).
- Restriction strategy (fixing of some variables temporarily, e.g., in linear programming).

Standard useful combinations of manipulations/strategies used in MODM are: dualisation, linearisation/relaxation, projection, outer linearisation/relaxation, e.g., cutting plane, projection/piece-wise, inner linearisation/restriction, e.g., Dantzig-Wolfe, projection/feasible directions or dualisation/feasible directions.

\subsection{MADM approach}

The 'best' design is selected among the discrete number of design alternatives via straightforward evaluation, as stated in Zanic et al. (1992). The increased speed of workstations provides an opportunity to model the complex DP as a multiple evaluation process by intentionally creating a large number of design variants. It is done by way of enumeration or random search methods as the simplest and most robust of non-gradient techniques. If sufficient density of non-dominated points is generated, one may obtain a 'discrete' inversion of the evaluation mapping for the most important parts of the design space. Therefore, it is possible to replace optimisation-oriented MODM approach with much simpler MADM. It implies generation, evaluation and filtering of non-dominated designs in the affine space for the final selection procedure in the metric space.

In this way, problems of discrete variables and disjoint domain, prohibiting application of most analytical methods, become solvable. MADM approach is particularly efficient in the CDP and in design of subsystems in the PDP.

\subsection{Design generation and evolution strategies}

Some MCDM methods and their combinations for the generation of good parent designs (e.g. on the non-dominated hyper-surfaces $\mathbf{X}^{N}, \mathbf{Y}^{N}$ or $\mathbf{M}^{N}$ ) are listed. Classical deterministic search methods (e.g. the Nelder-Mead simplex strategy used in MODM), may also be applied, particularly for exploration of the design space shape. Local and global search methods may differ and hybrid methods may emerge in the future. Six approaches of stochastic search are mentioned here, three of which are non-dominance driven, sequential and adaptive. The emerging computational paradigm is to follow processes in nature ('superb designer') and the last three methods are modelled accordingly. These methods are also more robust to local minima. Note that strategies (1)-(6) search from a population of points:

(1) Monte Carlo sampling in design space generates $n$ nondominated designs in $t$ trials. It can be used for start in strategies (2)-(6) and for multiple starting points in MODM. 
(2) Sequential adaptive generation of non-dominated designs (SAGeN) implies testing of feasible designs for the dominance in the Pareto sense. Non-dominated designs are used as centres of subspaces (mini-cubes) in the design space for further sequential ('chain') generation of non-dominated candidates for the final design selection, e.g., Zanic et al. (1992). Basic difference from strategy (1) are adaptive bounds as functions of current non-dominated point $x^{k}$. Fractional factorial designs (FFD) constructed from the Latin squares (Montgomery 2001) can be applied for efficient generation of designs and has proven efficient in higher cycles of adaptive design generation in subspaces around the non-dominated designs. The number of factor (variable, parameter) levels ranges from 2 to 5. Orthogonal arrays (e.g. L9, L27) with three levels accommodating up to 8 and 13 design variables are commonly used.

(3) Multi-objective genetic algorithms (MOGA) include (a) crossover, i.e., exchange of parts of chromosome contents (string of decimal or binary values of design variables $\mathbf{x}$ ), (b) mutation of chromosome content and (c) statistical selection of surviving designs. GA is modelled following natural selection with Darwinian survival of the fittest. They correspond to a randomised adaptive search. They differ from strategies (1) and (2) by recombination of the parameter set, not the parameters themselves. They use probabilistic and not deterministic transition rules regarding design fitness. The spectrum of MOGAs includes numerous operators that are listed, as given in Coello Coello (2007), according to their support of the primary MOGA goals:

(a) Preserve non-dominated points

- Dominance-based ranking - fitness assignment

- Non-Pareto versus Pareto approaches

- Archiving + elitism of chromosome population

(b) Progress towards points on Pareto front $\mathrm{PF}_{\text {true }}$

- Convergence to true computational Pareto front $\left(\mathrm{PF}_{\text {true }}\right)$

- Generating non-dominated phenotype points

- Explicit/Non-explicit building block manipulation

- Qualitative and quantitative performance metrics and visual comparisons

- Probabilistic multi-objective evolutionary algorithm (MOEA) models, local search incorporation etc.

(c) Maintain diversity of: points on $\mathrm{PF}_{\text {known }}$

- Diversity preservation

- Niching/fitness sharing and crowding on Pareto front (variations)

- Uniform/diverse non-dominated $\mathrm{PF}_{\text {known }}$

SPEA2 (strength Pareto evolutionary algorithm 2) and NSGA-II (non-dominated sorting algorithm II) are two of the most prominent MOEAs used when comparing the newly designed multi-objective optimisation methods due to their ability to achieve a high quality of a Pareto frontier on different types of optimisation problems.

NSGA-II (Srinivas and Deb 1994) builds a population of competing individuals, ranks, and sorts each individual according to the non-dominance level, applies evolutionary operations to create new pool of offspring, and then combines the parents and offspring before partitioning the new combined pool into fronts. NSGA-II then conducts niching by adding a crowding distance to each member. It uses this crowding distance in its selection operator to keep a diverse front by making sure each member stays a crowding distance apart. This keeps the population diverse and helps the algorithm to explore the fitness landscape.

SPEA2 (Zitzler et al. 2001) uses an external archive containing non-dominated solutions previously found (the so-called external non-dominated set). At each generation, non-dominated individuals are copied to the external nondominated set. For each individual in this external set, a strength value is computed. Fitness of each member of the current population is computed according to the strengths of all external non-dominated solutions that dominate it. The fitness assignment process of SPEA2 considers both the closeness to the true Pareto front and even distribution of solutions at the same time. A fine-grained fitness assignment strategy takes into account, for each individual, the number of individuals who dominate it and the number of individuals on which it dominates. It also uses a nearest-neighbour density estimation technique that guides the search more efficiently. Since the external non-dominated set participates in the selection process of SPEA, if its size grows too large, it might reduce the selection pressure, thus slowing down the search. Due to that, the authors have adopted a technique that prunes the contents of the external non-dominated set, while securing the preservation of boundary solutions, so that its size remains below a certain threshold:

(4) Evolution strategies (ES) (see, e.g., Michalewicz 1992) are similar to (3) but one of the major differences is that crossover/recombination is not very important, while the mutation is the primary operation for the evolution towards the optimal design.

(5) Simulated annealing is patterned on the physical process of the optimum layout of molecules due to annealing. The objective function of the optimisation problem is taken as the energy corresponding to a given system state (i.e. design). The design variations $\left(\mathbf{x}^{k}\right)$ for given 'temperature' (process control parameter) are treated as the probable states with the Boltzmann distribution. The number of random variations at each temperature and the rate at which temperature is lowered is called annealing schedule.

(6) Multi-objective particle swarm optimisation (MOPSO) is a relatively new optimisation technique inspired by the swarm behaviour where particles interact and fly 
to new positions based on their own knowledge and knowledge of the whole swarm. Although originally not intended for multi-objective optimisation, in the last few years, numerous algorithms have been developed, mostly using methods that were already used in MOGA.

Coello Coello and Lechuga (2002) proposed a method in which two repositories are maintained in addition to the search population. The first contained the global best individuals found so far by the search process, and the second contained a single local best for each member of the swarm (as in standard particle swarm optimization [PSO]). A truncated archive is used to store the (global) elite individuals. This archive uses the method from Knowles and Corne (2000) to separate the objective space (sub space of attribute space) into a number of hypercubes (an adaptive grid), where the more densely populated hypercubes have a lower score. The method also incorporates truncation of the most densely populated hypercubes when the archive exceeds its membership threshold.

Mostaghim and Teich (2003) proposed the sigma method with an objective of finding the best guides from the set of Pareto-optimal solutions, which is similar to compromise programming. The best guides for each particle are adopted to improve the convergence and diversity of a PSO. After the initial spread of particles, the first step is to calculate the value of sigma for each particle. In the multi-objective space, sigma is a vector of elements, where $n$ is the dimension of the objective space. To find the best global guide for each particle, it is necessary to fill out the archive with non-dominated solutions. Then, it is possible to calculate the distance between sigmas of dominated and non-dominated solutions. The global guide for the particle is selected as a non-dominated solution from the archive that has a minimum distance, which is calculated using the Euclidian norm. This procedure is repeated for all particles at each iteration.

Strategies (1)-(6) can be used in combination with the predictive task performed by meta-modelling techniques. Parallel processing is easily applicable to strategies (1)-(6), with the 'processor farming' (independent work) applied in the generation of feasible designs. The algorithmic parallelism of processors can speed up the process of filtration of non-dominated designs or the GA population selection.

Since strategies (3)-(6) are basically unconstrained optimisation techniques, the constraint set $\mathrm{g}(\mathrm{x})$ has to be included. Special procedures or penalty function approach,

$$
v^{P}(\mathbf{x})=v(\mathbf{x})+\mathrm{W}(\mathbf{g}(\mathbf{x})),
$$

are used. Penalty function $\mathrm{W}($.$) penalises the objective re-$ garding the level of constraint satisfaction. Immune network modelling (gene repair), with the antigen strings and generalist antibody strings, can also be used in strategy (3) to generate feasible 'children' (e.g. Yoo and Hajela 2001).
PSD and selection strategies in MADM imply simple and fast calculation of $\mathbf{L}^{N}$ for known $\mathbf{M}^{N}$. The minimisation problem is reduced to a simple comparison of $\mathbf{l}^{k}$ values.

\section{DP formulation for large-scale structural problems}

The design of complex thin-walled structures, such as ships, falls within the category of large-scale problems. Improvement in the design process efficiency for this type of problem can be accomplished by an application of appropriate simplifications/manipulations of the original problem, problem decomposition with a coordinated solution of a decomposed subproblem.

This section is devoted to the basics of these techniques. Methods considered are integrated into the interactive computing environment for the multi-criteria design (such as, OCTOPUS-Designer system) in order to enable effective and efficient interaction between the designer and the DP within the given design time frame.

\subsection{DP simplifications and manipulations for large-scale structural problems}

Simplifications come from the consideration of practical problems (contrary to the famous academic 'three bar truss club' optimisations).

Experience with large-scale structural optimisation problems, starting with Hughes et al. (1980), proves that the portion of compound failure surface contributed by each structural failure function is small and can be successfully linearised. Therefore, the envelope of feasible designs is transformed into a piece-wise linear failure surface (convex by definition). If the designer's objective functions are monotonic (only increasing or decreasing) with increase in structural scantlings, the optimal designs would lie on that surface. Functions for weight or cost of labour and material usually have this feature.

This practical reasoning opens up the possibility to use simple and efficient methods of operations research; see Hughes et al. (1980), Fleury (1989) or Rigo and Fleury (2001). Methods were further developed for large-scale optimisations tailored to naval architecture DeSS, e.g., MAESTRO (2012), OCTOPUS (2012), LBR5 (2012).

One of the most frequently used manipulations, especially for the structural problem, is dualisation, which combines objective function and constraint functions via Lagrange multipliers. They are the dual variables entering the problem linearly. Many practical and successful formulations are given in dual form. This formulation is especially efficient when the number of constraints is significantly larger than the number of design variables.

Other generally applicable manipulation and simplification strategies that are often used in engineering practice include meta-modelling or surrogate modelling, problem 
partitioning to the subsets of design variables and decomposition/coordination techniques.

\subsubsection{Surrogate modelling}

AMs of contemporary complex engineering systems, such as non-linear computational fluid dynamics (CFD) or FEM, can be computationally very demanding, and despite steady advances in computing power, the expense of running many analysis codes remains non-trivial. A single analysis of one design solution can take from minutes to hours or even much longer for, e.g., non-laminar and nonstationary 3D CFD problems. Due to these characteristics, as stated in the Introduction, the direct use of certain AMs is not possible in optimisation because optimisation demands several hundreds or even thousands of single analyses.

An application of surrogate modelling as an approximation (or surrogate) of expensive computer analysis codes can result in significant savings in both the number of analyses and the total time in which satisfactory optimal solutions are obtained. Another important aspect of a surrogate-based optimisation is the easier parallelisation of optimisation processes. Moreover, surrogates offer insight into the functional relationship between design parameters and design criteria, which is one of the obstacles in understanding the behaviour of numerical models.

The detailed overview of DOE methods for classical (physical) experiments is given in Montgomery (2001), while an overview of surrogate modelling for deterministic computer experiments (DACE - design and analysis of computer experiments) can be found in Simpson et al. (2001) or Fang et al. (2006).
The main difference between 'classical' and computer experiments is the non-existence of random errors for deterministic computer experiments, which leads to the conclusion that surrogate model adequacy is determined solely by systematic bias and that the classical notions of experimental blocking, replication and randomisation are irrelevant.

The steps necessary for the generation of surrogate models include: planning of experiments or sampling, execution of simulations with analysis modules, generation of surrogate models and validation of surrogate model adequacy. After selecting an appropriate experimental design according to the given criteria (Goel et al. 2008) and performing the necessary computer runs, the next step is to choose a surrogate technique and the corresponding fitting method. Many alternative methods exist, and there is no clear answer to which is better.

The selection of an appropriate surrogate method depends mostly on the characteristics of the physical phenomenon that is approximated. Some of the most frequently used surrogate models in engineering include: response surfaces (RS), kriging, radial basis functions (RBF), artificial neural network (ANN), support vector machine (SVM), splines (linear, cubic, non-uniform rational basis spline [NURBS]) and multivariate adaptive regression splines (MARS).

The first three of the above-mentioned surrogate modelling methods (RSM, RBF and kriging) are briefly described below. To test their usefulness for the modelling of structural responses, they are implemented in OCTOPUS (2012) (Designer) design support environment and used in Prebeg et al. (2012). Figure 3 presents a comparison of surrogate model errors for the variants of RSM, RBF and

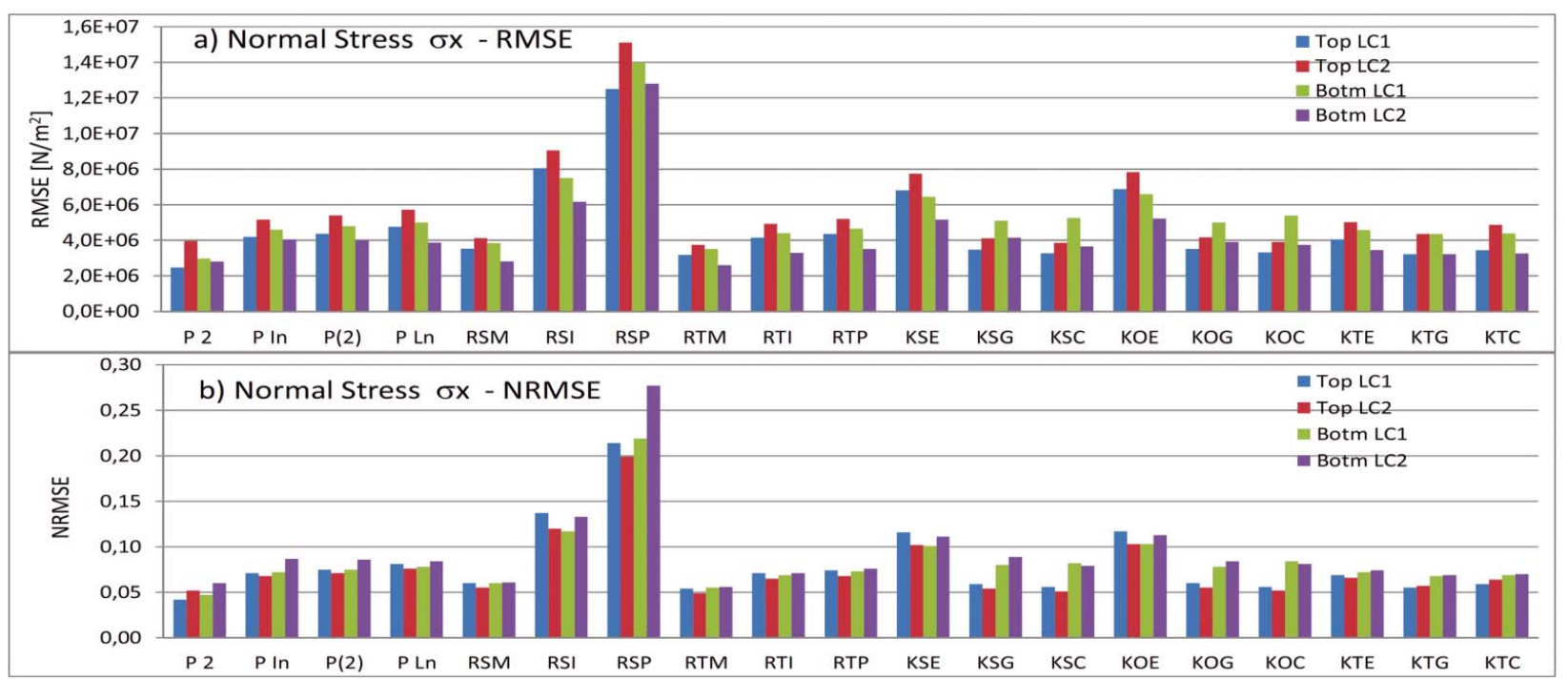

Figure 3. Surrogate model errors for the mean normal stress $\sigma_{x}$ in top/bottom substructures of a barge (Prebeg et al. 2012). (This figure is available in colour online.) 
kriging applied for the approximation of structural responses in that research.

Response surfaces (RS). This method is one of the most widely used surrogate methods in engineering practice. It approximates criteria functions using low-order polynomials, mostly simple linear and quadratic or some specific polynomials such as orthogonal Legendre polynomials. RS popularity for modelling of deterministic computer experiments, besides its good characteristics for certain type of problems, is due to the fact that surrogate modelling itself evolves from classical DOE theory where RS was used for the description of physical phenomena. Some of the applications in engineering includes: ship structural optimisation (see afore-mentioned study), Pareto front generation (Goel et al. 2007) etc.

Kriging. It is the surrogate modelling method that evolves from statistics, in this case geostatistics, where it was used to estimate mineral concentration over an area of interest, given a set of sampled sites from the area (Matheron 1963). Kriging model is a generalised linear regression model that accounts for the correlation in the residuals between the regression model and the observations. Kriging offers good flexibility to approximate many different and complex response functions and is a good choice for approximating deterministic computer models because it interpolates the observed data points. It has been used in a variety of applications, including conceptual design, structural optimisation, multi-disciplinary design optimisation and applications in aerospace engineering and mechanical engineering.

Radial basis functions (RBF). This surrogate method has been developed for scattered multivariate data interpolation (see, e.g., Powell 1992). The method uses linear combinations of a radially symmetric function based on the Euclidean distance or other such metric to approximate response functions. Like kriging, it is also an interpolationbased technique and is a good choice for approximating deterministic computer models.

\subsubsection{Decomposition and coordination}

Complex engineering systems can typically be considered as a hierarchy of coupled subsystems. The total performance of such complex systems is a combination of responses evaluated at each of the subsystems. A standard optimisation approach that does not take this behaviour into account usually treats the total system, or some part of the total system, as one integral element with one optimisation subproblem. Another approach is to treat the total DP as a group of non-coupled optimisation subproblems with their local objectives, not taking into account the influence that such changes have on the overall design, while system design objectives are not translated into subsystems/subproblems criteria. Decomposition of a complex system such as a ship can be done by an object decompo-

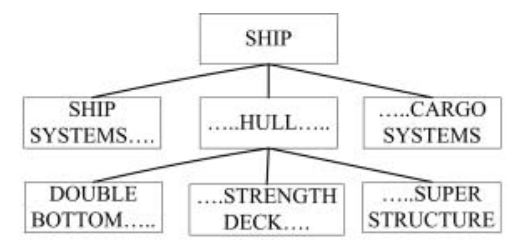

a) object decomposition

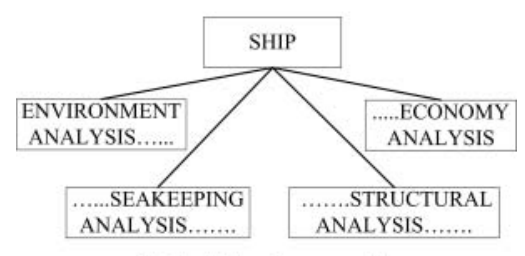

b) discipline decomposition

Figure 4. Two types of complex system decomposition.

sition or using discipline decomposition, as illustrated in Figure 4.

As stated in the overview by de Wit and van Keulen (2010), the field of multi-level optimisation and multidisciplinary optimisation is concerned with developing efficient analysis and optimisation techniques for complex systems that are made up of coupled subsystems (components). Unlike some other overviews that handled either multi-level optimisation or multi-disciplinary optimisation, the paper focuses on the general steps of methods that belong to either the field of multi-level optimisation or the field of multi-disciplinary optimisation.

Multi-level or multi-disciplinary optimisation methods rely on a decomposition of the optimisation problem into individual, yet coupled, optimisation problems. Thus, it is attempted to incorporate design variables, objectives and constraints originating from different levels and/or disciplines into the design.

According to de Wit and van Keulen (2010), the coordination approaches that handle decomposed problems, with respect to the discussion given above, can be classified according to Figure 5 .

\subsection{Software implementation of DeSS formulation}

Due to the complexity of the DeSS formulation for practical applications, it is convenient to develop a design environment that enables flexible implementation of the described process. A software architecture that implements the formulation given above for complex DeSS incorporates components: problem definition and solution modules ( $\boldsymbol{\Delta}$ and $\boldsymbol{\Sigma}$ ) and visualisation/ graphical user interface (GUI) modules $(\boldsymbol{\Gamma})$.

As an example of this unavoidable practical IT step, which is completing the applicable design procedure, the OCTOPUS (2012) is described. It involves combining analysis modules (MAESTRO and/or OCTOPUS-Analyzer) 


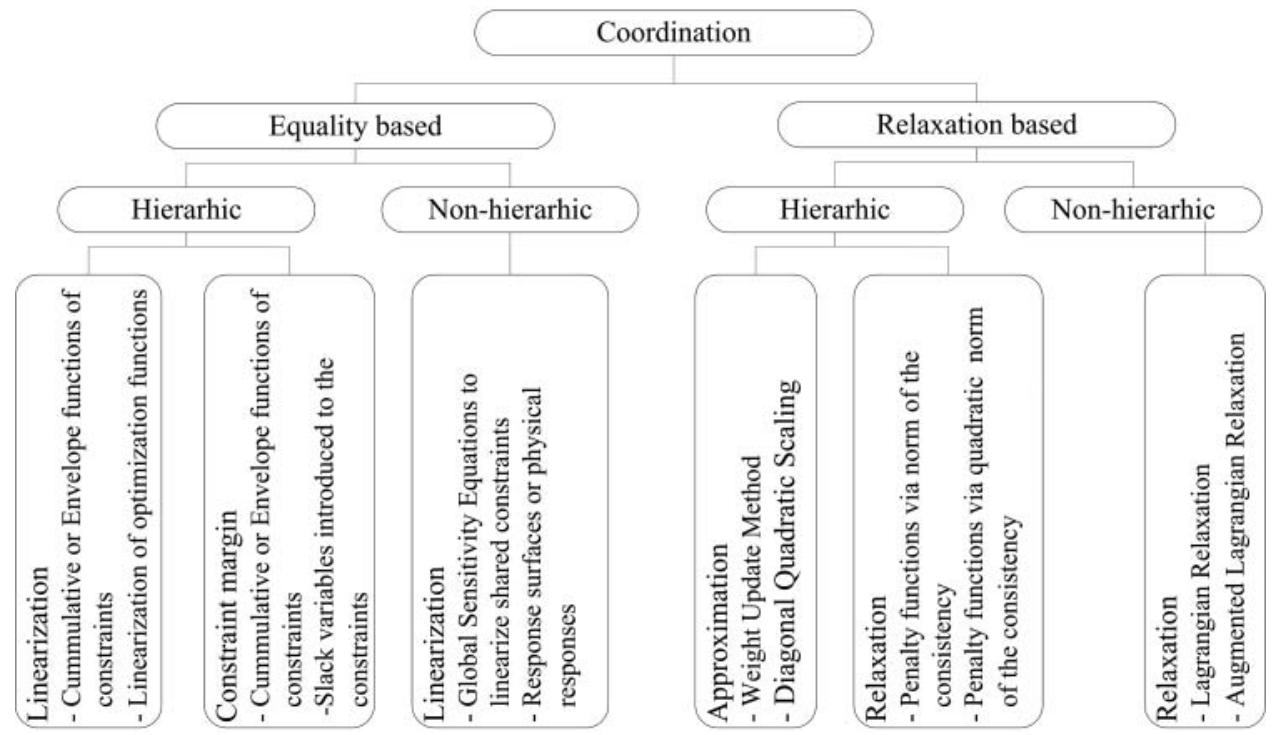

Figure 5. Classification of coordination approaches.

and OCTOPUS-Designer modules DeMak ( $\boldsymbol{\Delta}$ and $\boldsymbol{\Sigma}$ modules) and DeView ( $\boldsymbol{\Gamma}$ module) with a number of necessary IT components.

The work on DeMak started back in 1990 (see Zanic et al. 1993) but has recently been redefined to enable easy implementation of newly developed components, together with a flexible GUI that enables easy problem definition $(\Delta$ components) and problem-solving ( $\Sigma$ components) as well as visualisation of the output and the final design selection ( $\Gamma$ components). The concept of the framework that enables integration of analysis/simulation tools in the environment which has a collection of design support tools is similar to the concept of commercial software such as modeFRONTIER and ModelCenter ${ }^{\circledR}$. Although the framework is designed to allow flexible integration of general type of analysis modules, the tool has been tailored to match demands of general ship design (Zanic and Cudina 2009; Cudina et al. 2010) and structural ship design (Zanic et al. 2009).

The main components of OCTOPUS-Designer are DeMakGUI, DeMakMain and DeModel. DeMakGUI and DeMakMain are problem (i.e. AM)-independent. DeModel component wraps the given user model computation component $\boldsymbol{\Psi}$ (e.g. full MAESTRO code for structural problems). It gives the prescribed interface from the input in modules $\boldsymbol{\Phi}$ and $\boldsymbol{\varepsilon}$, for structure and loads, to the calculation modules in $\boldsymbol{\Psi}$. This enables communication between user model and user model-independent components. DeMakMain is the main component that encapsulates functionalities necessary for solving DeSS. More elaborate description of OCTOPUS-Designer architecture can be found in OCTOPUS (2012) (Designer) and Prebeg (2011).

\section{Applicable PSD procedure and a RoPax ship case study}

The overall goal of the design process is to simultaneously increase the ship-owner's profit and reduce shipyard production cost. Selected design parameters, having a significant effect on the design solution, have to be identified and discussed with the stakeholders as part of the DeSS formulated for the CDP and PDP, where the most far-reaching decisions are made. It is particularly demanding for the multi-deck ships characterised with the extensive superstructure whose influence on the primary strength has to be taken into account starting from the CDP. Topology/geometry-based optimisation can be assessed in the CDP; see Andric (2007), Zanic et al. (2007), while the PDP is used for efficient scantlings/material re-optimisations within the DeSS, including sensitivity/robustness assessment.

The complexity of synthesis procedure, shown through its dimensionality, non-linearity of response and feasibility models, stochastic definition of loading and of structural parameters, and subjectivity of quality assessments, permits only certain efficient combinations of calculation modules (matching current level of hardware developments).

The selection of advanced synthesis and analysis techniques has to be applied from the earliest stage of the design process on a concurrent basis (with respect to structure, production, operational performance and safety criteria). In the sequel, the procedures are presented for the CDPand PDP-streamlined DeSSs.

\subsection{Pareto supported design procedure applied to RoPax ship}

A practical structural design procedure/methodology, capable of embedding multiple design quality criteria, is 


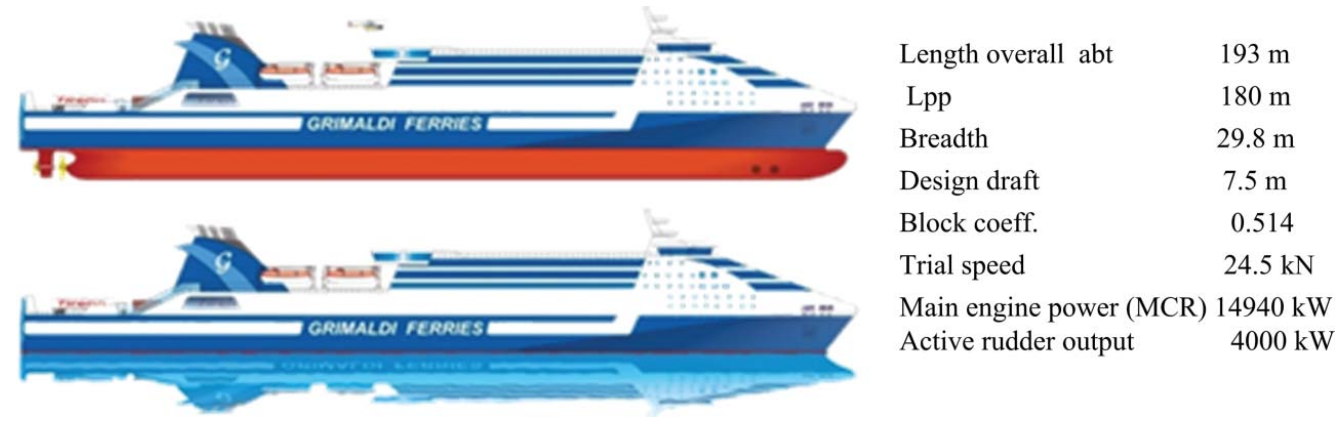

Figure 6. Main characteristics of a new RoPax ship. (This figure is available in colour online.)

presented through the benchmark case study on the innovative RoPax ship; see Figure 6.

Ropax ship design was part of the EU FP6 project IMPROVE (see ISSC 2009; Rigo et al. 2010; Zanic, Andric, et al. 2010) and included a multi-stakeholder approach involving the Grimaldi Group (owner), Uljanik (shipyard) and $\mathrm{BV}$ as a Classification Society. For this case, the modules inbuilt in OCTOPUS/MAESTRO software (see Table 1) were used.
The proposed flexible methodology (see Figure 7) tailored to the RoPax ship model combines three design steps divided into design blocks (subproblems: optimisation/selection) for the CDP and PDP. Models used in the case study are given in Table 1 .

The generic design block template, applicable in formulation of any of design subproblem, is presented in Figure 8 (with examples for particular items). Explanation of the basic taxonomy is given in the Editorial.

\begin{tabular}{|c|c|c|}
\hline CDP & Step 1/Blocks 1-2 & topology, geometry, scantling optimization) \\
\cline { 2 - 3 } & Step 2/Blocks 3-6 & concept exploration: e.g. safety as one of objectives \\
\hline PDP & Step 3/Block 7 & scantling re-optimization of preferred design \\
\cline { 2 - 3 } & Step 3/Block 8 & final analysis with all available software \\
\hline
\end{tabular}

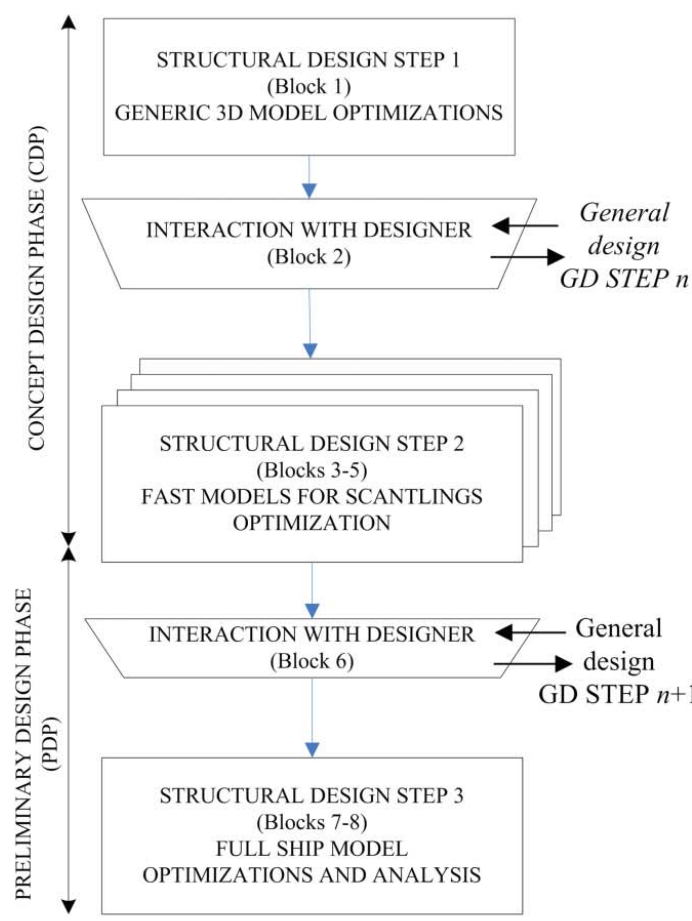

Legend: GD-general design, SD-structural design

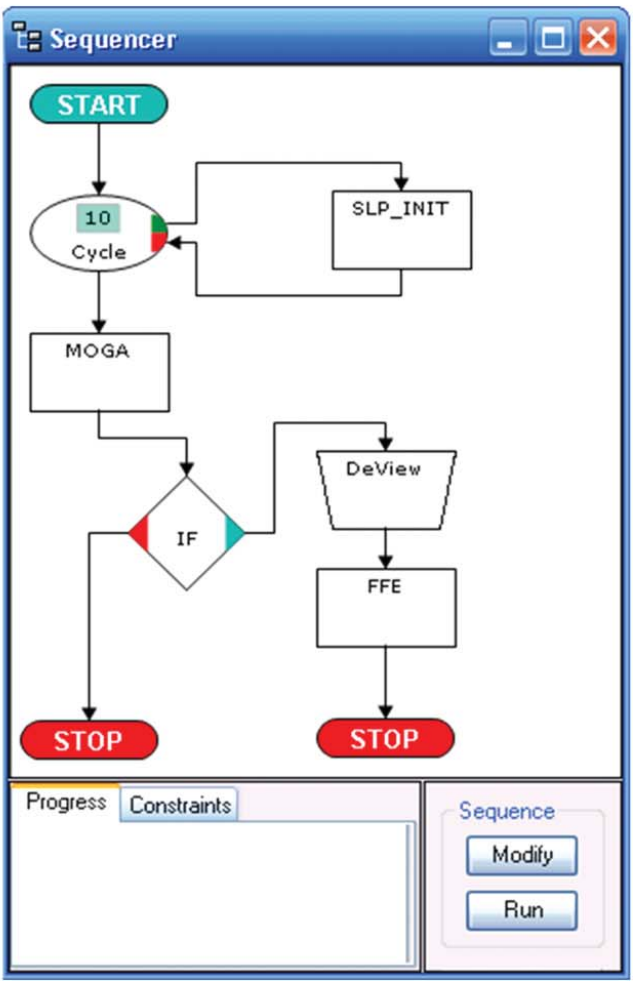

Figure 7. Design support problem sequence diagram (automatic sequencer is part of OCTOPUS-Designer). (This figure is available in colour online.) 


\begin{tabular}{|c|c|c|c|}
\hline \multicolumn{4}{|c|}{ GENERIC OPTIMIZATION BLOCK (taxonomy: see Editorial; IT-modules: see Table 1) } \\
\hline \multicolumn{4}{|c|}{ BLOCK TITLE-User given and PROBLEM FORMULATION ID } \\
\hline ITEM (n-tuple) & \multicolumn{3}{|c|}{ MODULES $\left(\right.$ e.g. $\Sigma^{\text {MOGA }}$ ) or FORMULAE (e.g. $\boldsymbol{a}^{\text {TTS DEFLECTIONS }}$ ) or DATA SET $\left(\right.$ e.g. $x^{\mathrm{GI}}$ ) } \\
\hline Variables $(\mathbf{x})$ & {$\left[\mathbf{x}^{\mathrm{G}} \mathbf{x}^{\mathrm{T}} \mathbf{d}^{\mathrm{S}} \mathbf{d}^{\mathrm{M}}\right]$} & (descriptors-obligatory), & \multirow{11}{*}{ Phase ID } \\
\hline Parameters $(\mathbf{z})$ & & (module name $\Phi, F, \Phi^{\text {IACS Rule min })}$ & \\
\hline Attributes $(\mathbf{y})$ & $\Omega^{(\max ) \text { NAME }}$ & [available module NAME, e.g. from Table 1)], & \\
\hline Objectives (o) & & for objective: specify direction (min-max) & \\
\hline Constraints (g) & $\alpha^{\text {SAFETY-NAME: }}$ & [available module NAME e.g from Table 1)] & \\
\hline$\alpha(\mathrm{g})>\mathrm{g}_{\min }$ & $\alpha^{\text {SHIP-TEXT: }}$ & [TEXT, e.g. Global constr. or ShipGA or Deflection limits, etc.? & \\
\hline Analysis & $\varepsilon^{\text {LOADS TEXT: }}$ & [ TEXT, e.g. Long. Strength- LC $_{1}$ or Racking BV-LC 2 ?; & \\
\hline Modules (AM) & $\rho^{\text {RESPONSE SOLVER NAME }}$ & [NAME e.g. OCTOPUS or MAESTRO] & \\
\hline Synthesis & $\Sigma^{\text {OPTIMIZ. SOLVER NAME: }}$ & [available module NAME e.g. SLP or ES (MCS, FFE, DOE, & \\
\hline Modules (SM) & & OA+ANOVA) or MOGA or MOPSO, etc.)] & \\
\hline Results & $\Gamma^{\mathrm{TEXT}}:$ & [TEXT e.g. Pareto designs, preferred designs, fuzzy functions, $L_{p}$ & \\
\hline
\end{tabular}

Figure 8. Description of generic optimisation block.

Selected combination of design blocks is given in Figure 9 for the CDP and in Figure 10 for the PDP in format (description [x, y, g, AM, SM], design model, results) and is discussed in the sequel.

A sequence of well-defined design subproblems, as efficiently defined by design blocks, can be conveniently modified/extended to suit designer's ideas/needs and implemented within the developed design environment.

Three basic design steps, covered by the example, are further defined as:

(1) Step 1-CDP; see Figure 9: Analysis and optimisation of ship model variants specified by the general designer-specified ship model variants. The structural design is based on the generic ship 3D FEM models, of the CDP fidelity level, e.g. using gross elements/surrogates (see Andric and Zanic 2010) and analytically defined adequacy criteria. Loads and adequacy criteria are based on the BV Classification Society Rules. It is performed as the first design task (Block 1).

Optimisation of different design concepts for given objectives (cost, mass etc.) with respect to the topological, geometrical and scantling variables enables their fair comparison. In the context of general design, the designer's selection (PSD) of generated design variants should be performed as the second design task (Block 2 ), using the global design quality measures on the grid of optimised variants. Preferred design(s) may proceed to Step 2.

(2) Step 2-CDP; see Figure 9: Control substructures (e.g. bays) of different ship segments could be modelled, using the computationally very fast models of the CDP fidelity, e.g. 2.5D models (Zanic et al. 2007), for generation of design alternatives on the Pareto frontier (Blocks 3-5). They can be further validated using the developed adequacy and quality measures (safety, vibration, fatigue, robustness and production cost).

A life cycle cost (LCC) module can be used in determination of the optimal combination of different substructures, as starting points for the next PDP; see Zanic, Andric, et al. (2010). In the context of general design, the designer's subjective selection of generated improved design variants (PSD) is performed using the global design quality measures on the grid of the optimised substructure variants (Block 6).

(3) Step 3-PDP; see Figure 10: The full-ship 3D FEM model should be developed (with convenient class required mesh size) to generate the optimal design variant using safety, weight, cost and fatigue criteria and based mostly on scantlings optimisation (Block 7). For the final design validation and generation of the final design for the class approval, the full-ship 3D FEM model should have standardised scantlings/parameters (on PDP fidelity). Direct wave load calculations could, if possible, be performed and applied in generation of design loads. Safety analysis should be performed with the utmost care. Vibrations and noise validation should be also performed (Block 8). If key performance indicators (KPIs) are unsatisfactory, return to Block 7 is needed.

\subsubsection{Discussion of the case study results}

Application of the developed design procedure resulted in an optimal structural design of RoPax with two superstructure decks, optimal parking area on lower decks, vertical center of gravity (VCG) position etc., combined with minimisation of the ship lightweight and related savings in fuel and other operational costs. Overall results, achieved for each design step, are given as a table in Figure 10 (last row/last column). A summary of the obtained results is given in the following sequel: 

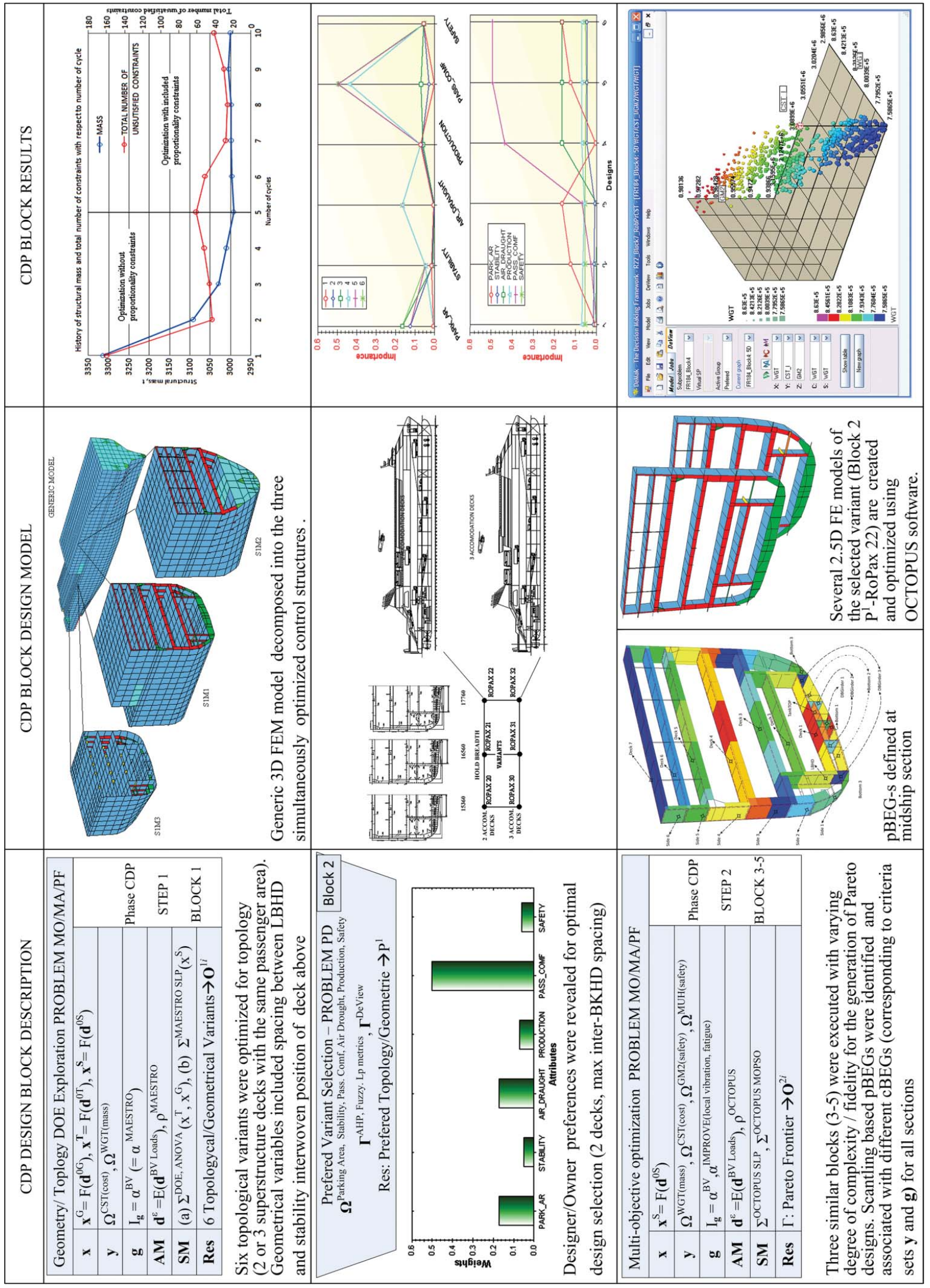


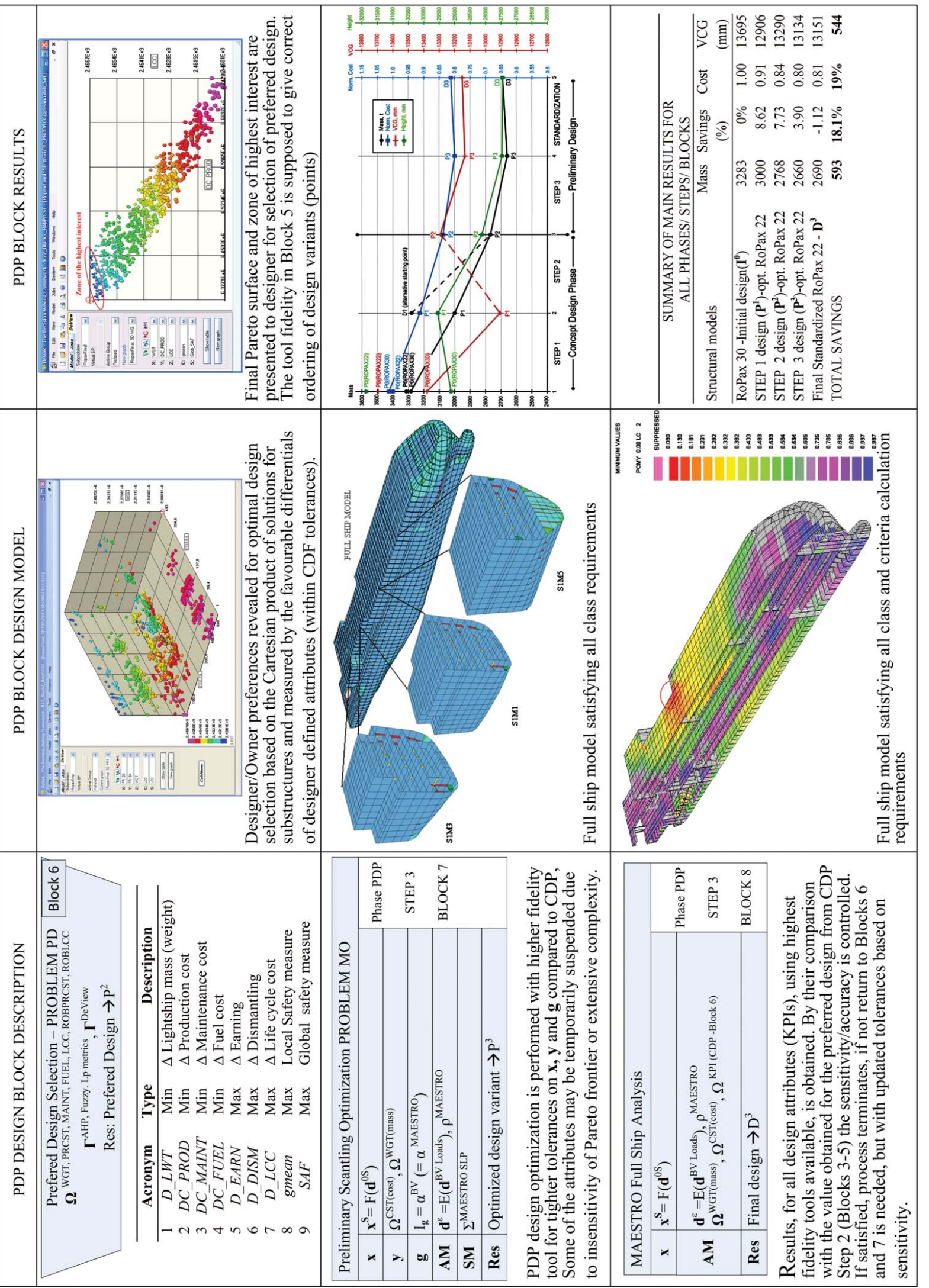


- STEP 1 design: Structural optimisation resulted in a decrease in the structural mass of 283 tonnes $(8.62 \%)$ with respect to the initial structural mass of prototype $\mathbf{I}^{\mathbf{0}}$ (RoPax 30). It is denoted as $\mathbf{P}^{\mathbf{1}}$, obtained using PSD formulation.

- STEP 2 design: The starting point of the second design step was $\mathbf{P}^{\mathbf{1}}$. Multi-criteria optimisation during the second design step resulted in a structural mass decrease $\left(\mathbf{P}^{2}\right)$ of 232 tonnes (7.7\%) with respect to the $\mathbf{P}^{1}$ solution.

- STEP 3 design: Structural optimisation resulted in a structural mass decrease of 108 tonnes (3.9\%) with respect to the structural mass of the $\mathbf{P}^{2}$ solution, denoted as $\mathbf{P}^{3}$. At the end of the design sequence, structural scantlings were standardised and the final $\mathbf{D}^{3}$ solution was generated. Due to standardisation, the structural mass with respect to the $\mathbf{P}^{\mathbf{3}}$ solution is slightly higher for 37 tonnes $(1.12 \%)$.

Finally, the full ship had $6.97 \%$ less lightship weight in comparison with the prototype ship. The gain of 5\% more trailer lanes (cargo capacity) on the tank top was achieved by investigating different positions of longitudinal ballast tank bulkhead (constrained with respect to damage stability calculation) and at the same time the ballast volume was minimised.

Overall savings, from prototype RoPax 30 to the proposed (standardised) design $\mathbf{D}^{\mathbf{3}}$, are very challenging: $18 \%$ in weight, $19 \%$ in cost and $4475 \mathrm{~mm}$ in ship height (one less tier of superstructure was proposed). It, however, proves that the cascade of optimisation steps in the novel design procedure produced very satisfactory results. They were further cross-checked with the 3D FEM full-ship analysis on the class-required fidelity level. Quality of the RoPax project has been significantly increased due to the reduction in the fuel consumption (better propulsion and ship hull form, reduced weight etc.), increase in payload (larger parking area) and better LCC performance.

It is also very important to acknowledge that the reduction in fuel consumption has significantly reduced $\mathrm{CO}_{2}$ emission, thus increasing environmental friendliness and also ensuring that requirements related to pollution would be satisfied in the future.

Also, the developed methodology gives yards and owners a possibility to select competitive design solutions by the following paradigm: better ship for the yard production and more profitable ship for the owner.

The presented modern RoPax design and its design attribute values prove gains from such double competitiveness, as well as the efficiency of the methodology that was driving the design process.

\subsection{Influence of safety as an objective on structural design (sensitivity study)}

To support the arguments on the potential gains when safety measures are taken as objectives in the CDP, a structural concept exploration of the multi-step multi-criteria procedure, the results of Blocks 3-5 were scrutinised.

DeSS is applied to the benchmark example of the RoPax ship (Zanic, Andric, et al. 2010; Zanic, Kitarovic, et al. 2010). CDP level calculation was performed using structural weight and simplified safety measures to improve given preferred design obtained in topology/geometry optimisation of the CDP.

Design procedure steps were executed using a fast and balanced collection of analysis and synthesis modules/methods of the OCTOPUS/MAESTRO design system (Table 1 and Figure 7). For each generated design, the AMs/analysis modules $\rho, \alpha, \Omega$ were used for calculation of:

(1) ship response fields $\sigma^{\mathbf{L C}}$ (due to primary strength and racking-based load cases),

(2) ship ultimate longitudinal strength $\left(M_{\text {ult }}\right)$,

(3) serviceability and collapse safety criteria on gross panels $\mathbf{g}^{\mathrm{SAF}}=\left\{g_{\mathrm{SAF} i}\right\} ; i=1, n_{\text {crit }}$.

Examples included approximately 15,000 safety checks for eight load cases; 130 stiffened panels and 15 failure modes per each panel were used for calculating compound safety measures. BV Rules loading and respective load factors were applied.

Safety measures, $\mathbf{g}^{\mathrm{SAF}}\left\{\mathrm{GM} 1, \mathrm{GM} 2, g_{<0.05}, g_{\min 5 \%}\right\}$, as examples, are given below:

$$
\begin{aligned}
\mathrm{GM} 1 & =g_{\min }=\min \left\{g_{\mathrm{SAF} i}\right\} ; \mathrm{GM} 2=g_{\text {mean }} \\
= & \frac{\sum_{i=1}^{n_{\text {crit }}} u_{i}\left(g_{\mathrm{SAF} i}\right)}{n_{\text {crit }}} ; g_{<0.05}=\left.\sum_{i=1}^{n_{p}} \sum_{j=1}^{n_{c}} g_{i j}\right|_{<0.05} ; \\
g_{\min 5 \%}= & \frac{\left.\sum_{i=1}^{n_{5} \%} g_{i j}\right|_{5 \%}}{n_{5 \%}} ;\left.g_{i j}\right|_{5 \%}(\text { worst } 5 \%) ;
\end{aligned}
$$

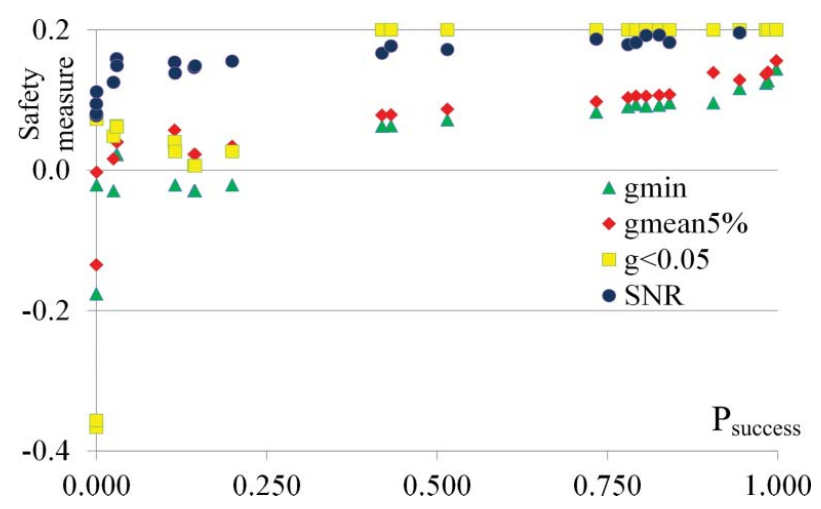

Figure 11. Comparison of different approximate safety measures with CALREL-calculated Psuccess as abscissa. (This figure is available in colour online.) 


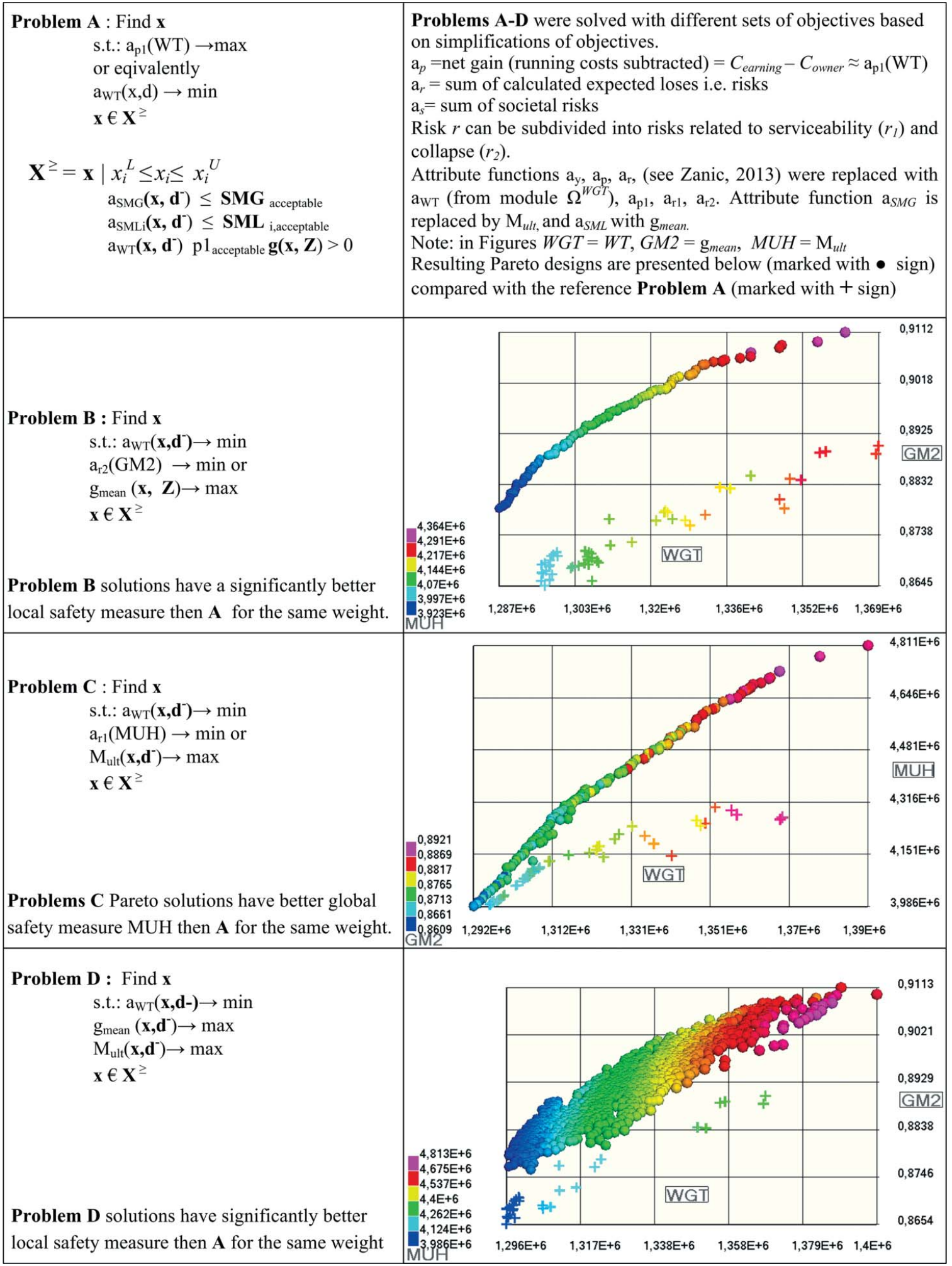

Figure 12. Influence of safety as design objective on the design performance. (This figure is available in colour online.) 
where $g_{\mathrm{SAF} i}$ is normalised safety factor value of BVspecified criterion $i$ and $u_{i}()$ is a fuzzy function signifying the importance of the constraint value for the safety of the panel.

RBD-based calculation was performed using CALREL code (Liu et al. 1992) for the typical stiffened deck panel in the midship region, based on wave-induced hydrodynamic load calculation. Statistical data (distributions, means, covariances [COVs] etc.) were obtained for all load cases and all load components. System reliability option in the CALREL software was applied.

From Figure 11, we see that safety measure $g_{\text {mean }}, 5 \%$ can be used as local design objective, provided that panel integrity is maintained by the request on the normalised adequacy factor $g()>-0.05$ for all load cases and all failure modes. Taguchi has developed a signal-to-noise ratio (SNR) to provide a way of measuring robustness; see Taguchi (1986), Phadke (1989) and Ross (1988).

DP formulations regarding safety as an objective (left column) and achieved results (right column) are presented in Figure 12. Those designs with their problem formulation are discussed in more details in Zanic, Kitarovic, et al. (2010) and Zanic (2013).

Problem A (basic, single objective reference design) presents the minimum weight designs generated through iterations, implying that the final minimum weight design is presented together with other intermediate results. Those designs are marked as 3D stars (visible as ' + ' sign).

Presented designs for the Problems $B-D$ (contrasted to design A, i.e., standard minimal weight design) are only the final set of non-dominated designs (Pareto frontier) and are presented by spheres.

Problem B introduces a local safety measure SML (i.e. GM2 obtained from function $g_{\text {mean }}$ ) as an additional objective.

Problem $C$ introduces a global safety measure SMG (MUH obtained from function $M_{\text {ult }}$ ) as an additional objective.

Problem D (using PSD) introduces both local safety measure GM2 and global safety measure MUH as additional objectives (colour-coded in Figure 12, cells 4, 2). Since the Pareto frontier in this case is spanned by the three objectives, it is no longer a curved line as in Problems $B$ and $C$, but a surface, so its projection has a range of points for the specified weight contour. This cell clearly demonstrates that Problem $D$ solutions have significantly better local safety measure then Problem $A$ solutions for the same weight.

Finally, investigating all comparisons (Problem $B$ vs. $A, C$ vs. $A$ and $D$ vs. $A$ ), the following conclusions can be made:

- Safety measures used as objectives serve as powerful independent attractors towards the parts of the attribute space where a better safety is achieved for the same weight.

- Another beneficial characteristic is a more reliable convergence to the global minimum weight design. This is due to the fact that optimisation algorithms advance to the global minimum weight design from the designs that have the best achieved safety for the given weight. In the case of structural design, this also means that the 'distance' from constraints (failure surfaces) is greatest for a given weight.

- Additionally, local and global safety measures are not directly coupled since the same level of local safety can be obtained with different degrees of global safety and vice versa $\rightarrow$ both measures should be used simultaneously.

The basic conclusion of this additional design step is that safety considerations have to be included, not only as design constraints, but also as a design goal, if we want to obtain the maximum gain for the given investment from the owners' and societal point of view.

\section{Conclusions}

This paper is a complementary to the Editorial of this issue of the Journal and presents in more detail the methods and techniques with their application to the practical complex ship structure-design example.

The design support methodology (techniques and procedures) for multi-criteria synthesis of large thin-walled ship structures in concept and preliminary design is presented with the following main conclusions:

- To decrease the design process time, the optimisation process has to follow the designer's data availability and provide fast answers with adjusted models. This rules out standard optimisation procedures as inoperable and requires the development of a refined approach to the multi-criteria, multi-stakeholder DP in the form of a DeSS with flexible and fully interactive design environment tailored to large-scale problems.

- Improvement in the design process efficiency for complex thin-walled ship structures can be accomplished by an application of appropriate simplifications/manipulations of the original problem, by problem decomposition with the coordinated solution of decomposed subproblems and by an application of appropriate multi-criteria decision-making techniques.

- An efficient multi-step procedure is also needed in order to solve the complex topology optimisation problem (with interwoven scantling/geometry optimisation). 
- The presented approach combines three design steps for the fast generation of design variants regarding topological, geometrical and scantlings variables. As a case study, it was successfully implemented for the new RoPax ship, where a significant improvement has been achieved in structural and general design aspects.

- One of the major drivers of the presented achievements is the design methodology that has closely joined two collaborating design systems (general ship design and structural design) as well as basic stakeholders (owner, yard/designers, regulatory institutions) by way of formulation of the DP for rational decision-making within the PSD paradigm. It has been successfully tested in interactive work with stakeholders, revealing their subjective preferences in the interactive graphic environment (using, e.g., OCTOPUS software) and making quality decisions possible.

- A case study also confirmed that the competence of structural subsystem designers is to generate the Pareto frontier of non-dominated structural designs. It is then used for a higher-level decision-making to multiply the benefits from subsystem gains within the general DeSS approach.

Finally, a complex yard-owner-society relation can be established using simplified models, leading to satisfaction of the DP multiple objectives:

(1) Better ship for the owner and better ship for the yard (demonstrated in the EU FP6 IMPROVE project (Rigo et al. 2010).

(2) Maximum gain for the given investment from the owners' and societal point of view. It is achieved if we include safety considerations also as an objective (as demonstrated above).

Both considerations may be satisfied by using the PSD techniques within the modern DeSS.

\section{Acknowledgements}

Thanks are due to the long-term support of the Croatian Ministry of Science, Education and Sports: project 120-1201829-1671. The European Commission under the FP6 Sustainable Surface Transport Program, STREP project IMPROVE (www.improveproject.eu), contract no. FP6-031382 also supported a part of the study presented in the paper. Thanks are due to members of the OCTOPUS group (www.fsb.hr/octopus), to the design teams of the ULJANIK shipyard (www.uljanik.hr) and Navis Consult d.o.o., Rijeka-Part of Rolls-Royce Marine (www.rolls-royce.com/ marine). Finally, authors would like to express special thanks to friends and colleagues at the Advanced Marine Technology Centre, DRS Technologies, Inc. (www.drs-ds.com), Parsippany, NJ,
USA, on the excellent work on the MAESTRO software development for so many years.

\section{Abbreviations:}

\begin{tabular}{|c|c|}
\hline AM & Analysis model \\
\hline ANN & Artificial neural network \\
\hline $\mathrm{BC}$ & Boundary conditions \\
\hline BV & Bureau Veritas \\
\hline CSR & Common structural rules \\
\hline CDP & Concept design phase \\
\hline DACE & Design and analysis of computer experiments \\
\hline DeSS & Design support system \\
\hline DOE & Design of experiments \\
\hline DDP & Detail design phase \\
\hline DSP & Decision support problem \\
\hline FEM & Finite-element (FE) method \\
\hline FFE & Fraction factorial experiments \\
\hline GA & General arrangement \\
\hline GBS & Goal-based standards \\
\hline GUI & Graphical user interface \\
\hline IACS & $\begin{array}{l}\text { International Association of Classification } \\
\text { Societies }\end{array}$ \\
\hline IMO & International Maritime Organization \\
\hline ISSC & International Ship Structure Committee \\
\hline KPI & Key performance indicator \\
\hline $\mathrm{LCC}$ & Life cycle cost \\
\hline MADM & Multi-attribute decision-making \\
\hline MAESTRO & 3D FE structural design software \\
\hline MCDM & Multi-criteria decision-making \\
\hline MODM & Multi-objective decision-making \\
\hline MOGA & Multi-objective genetic algorithms \\
\hline MOPSO & Multi-objective particle swarm optimisation \\
\hline MUS & $\begin{array}{l}\text { Ultimate hull girder vertical bending moment } \\
\text { in sagging condition }\end{array}$ \\
\hline MUH & $\begin{array}{l}\text { Ultimate hull girder vertical bending moment } \\
\text { in hogging condition }\end{array}$ \\
\hline OCTOPUS & $\begin{array}{l}\text { Integrated ship structural modelling, analysis } \\
\text { and design support system, developed at the } \\
\text { University of Zagreb }\end{array}$ \\
\hline PDP & Preliminary design phase \\
\hline PSD & Pareto supported decision-making \\
\hline RBD & Reliability-based design \\
\hline $\mathrm{RBF}$ & Radial basic functions \\
\hline RS & Response surface \\
\hline SLP & Sequential linear programming \\
\hline SM & Synthesis model \\
\hline SVM & Support vector machine \\
\hline VCG & Vertical centre of gravity \\
\hline
\end{tabular}

\section{References}

Andric J. 2007. Decision support methodology for concept design of the ship structures with hull-superstructure interaction [PhD dissertation (in Croatian)]. [Zagreb (Croatia)]: University of Zagreb. 
Andric J, Zanic V. 2010. The global structural response model for multi-deck ships in concept design phase. Ocean Eng. 37:688-704.

Coello Coello CA, Lamont GB, Van Valdhuizen DA. 2007. Evolutionary algorithms for solving multi-objective problems. New York: Springer.

Coello Coello CA, Lechuga MS. 2002. MOPSO: a proposal for multiple objective particle swarm optimization. Proceedings of Congress on Evolutionary Computation, CEC '02; 2002 May 12-17; Honolulu, HI. New York: IEEE Press. p. $1051-1056$.

Cudina P, Zanic V, Prebeg P. 2010. Multiattribute decision making methodology in the concept design of tankers and bulkcarriers. Proceedings of PRADS 2010; 2010 Sep 19-24; Rio de Janeiro, Brazil.

de Wit AJ, van Keulen F. 2010. Overview of methods for multilevel and/or multi-disciplinary optimization. Proceedings of the 51th AIAA/ASME/ASCE/AHS/ASC Structures, Structural Dynamics, \& Materials Conference; 2010 Apr 12-15; Orlando, FL. Reston, VA: AIAA.

Fang K, Li R, Sudjianto A. 2006. Design and modeling for computer experiments. London: Chapman \& Hall.

Fleury C. 1989. CONLIN, an efficient dual optimizer based on convex approximation concepts. Struct Optimiz. 1:81-89

Goel T, Haftka RT, Shyy W, Watson LT. 2008. Pitfalls of using a single criterion for selecting experimental designs. Int $\mathrm{J}$ Numer Meth Engng. 75:127-155.

Goel T, Vaidyanathan R, Haftka R, Shyy W, Queipo N, Tucker K. 2007. Response surface approximation of Pareto optimal front in multi-objective optimization. Comput Methods Appl Mech Eng. 196(4-6):879-893.

Holmberg T, Hunter SD. 2011. Increasing efficiency in the ship structural design process. Proceedings of the 10th International Conference on Computer and IT Applications in the Maritime Industries, COMPIT'11; 2011 May 2-4; Berlin, Germany, Hamburg: Technische Universität Hamburg-Harburg. p. 536-550.

Hughes OF, Paik JK. 2010. Ship structural analysis and design. New Jersey, USA: The Society of Naval Architects and Marine Engineers.

Hughes OF, Mistree F, Zanic V. 1980. A practical method for the rational design of ship structures. J Ship Res. 24(2):101-113.

IACS. 2010. Common Structural Rules for Double Hull Oil Tankers; July 2010; International Association of Classification Societies.

IACS. 2008. Common Structural Rules for Bulk Carriers; July 2008; International Association of Classification Societies.

ISSC. 2009. Report of Committee IV.1. Proceedings of International Ships and Offshore Structures Congress; 2009 Aug 16-21; Seoul National University, Seoul, Korea: STG

Knowles JD, Corne DW. 2000. Approximating the nondominated front using the Pareto archived evolution strategy. Evol Comput. 8(2):149-172.

LBR5. 2012. Documentation. Liege (Belgium): ANAST Laboratory, University of Liege.

Liu PL, Lin HZ, Der Kiureghian A. 1992. CalREL user manual. Berkeley (CA): Department of Civil Engineering, University of California at Berkeley

LS-INGRID user's manual. 1998. Livermore (CA): Livermore Software Technology Corporation.

MAESTRO. 2012. Documentation ver. 10.0, DRS Defense Solutions, LLC. Stevensville (MD): Advanced Marine Technology Center (AMTC).

Matheron G. 1963. Principles of geostatistics. Econ Geol. 58(8):1246-1266
Michalewicz Z. 1992. Genetic algorithms + data structures = evolution programs. Berlin (Germany): Springer-Verlag.

Montgomery DC. 2001. Design and analysis of experiments. 3rd ed. New York: John Wiley.

Mostaghim S, Teich J. 2003. Strategies for finding good local guides in multi-objective particle swarm optimization (MOPSO). Proceedings of Swarm Intelligence Symposium SIS '03; 2003 Apr 26; Indianapolis, IN, New York: IEEE Press. p. 26-33.

OCTOPUS. 2012. Octopus-Designer and Octopus-Analyzer manuals. Zagreb (Croatia): University of Zagreb, Faculty of Mechanical Engineering and Naval Architecture.

Paik JK, Thayamballi AK. 2003. Ultimate limit state design of steel-plated structures. Chichester: John Wiley.

Phadke SM. 1989. Quality engineering using robust design. Englewood Cliffs (NJ): Prentice Hall.

Powell MJD. 1992. The theory of radial basis functions approximation in 1990. In: Light WA, editor. Advances in numerical analysis volume II wavelets subdivision algorithms and radial basis functions. Oxford: Oxford University Press. p. 105-210.

Prebeg P. 2011, Multi-criteria design of complex thin-walled structures $[\mathrm{PhD}$ dissertation (in Croatian)]. [Zagreb (Croatia)]: University of Zagreb.

Prebeg P, Zanic V, Vazic B. 2012. An application of complex system optimization techniques to the ship structural design. Proceedings of the 11th International Marine Design Conference; 2012 Jun 11-14; Glasgow, United Kingdom.

Rigo P. 2001. A module-oriented optimization tool. Proceedings of the Eighth International Symposium on Practical Design of Ships and Other Floating Structures, PRADS 2001; 2001 Sep 16-21; Shanghai, China. p. 51-58.

Rigo P, Fleury C. 2001. Scantling optimization based on convex linearizations and dual approach - Part I and II. Mar Struct. 14:631-649.

Rigo P, Zanic V, Ehlers S, Andric J. 2010. Design of innovative ship concepts using an integrated decision support system for ship production and operation. Brodogradnja. 61(4): 367-381.

Ross PJ. 1988. Taguchi techniques for quality engineering. New York: McGraw-Hill.

Simpson TW, Peplinski JD, Koch PN, Allen JK. 2001. Metamodels for computer-based engineering design. Eng Comput. 17(2):129-150.

Srinivas N, Deb K. 1994. Muiltiobjective optimization using nondominated sorting in genetic algorithms. Evol Comput. 2(3):221-248.

Taguchi G. 1986. Introduction to quality engineering. Dearborn (MI): Asian Productivity Organization (distributed by American Supplier Institute Inc.)

Yoo J, Hajela P. 2001. Fuzzy multi-criterion design using immune network simulation. Struct Multidiscipl Optimiz. 22:188-197.

Yu P-L. 1985. Multiple criteria decision making. New York: Plenum Press.

Zanic V. 2013. Methods and concepts for the multi-criteria synthesis of ship structures. Editorial [this Special issue]. 8(2).

Zanic V, Andric J., Prebeg P. 2007. Decision support methodology for concept design of multi-deck ship structures. Proceedings of PRADS 2007; 2007 May 31-Jun 5; Houston, TX, Houston, TX: ABS

Zanic V, Andric J, Prebeg P. 2009. Design environment for structural design: application to modern multi-deck ships. Proc IMechE, part M. 223(M1):105-120.

Zanic V, Andric J, Prebeg P, Stipcevic M, Piric K. 2010. RoPax structural design - multi-level decision support methodology. Proceedings of PRADS; 2010; 2010 Sep 19-24; Rio de 
Janeiro, Brazil. Rio de Janeiro, Brazil: Aped-Apoio e Producao Ltda.

Zanic V, Cudina P. 2009. Multiattribute decision making methodology in the concept design of tankers and bulk carriers. Brodogradnja. 60(1):19-43.

Zanic V, Das PK, Pu Y, Faulkner D. 1993. Multiple criteria synthesis techniques applied to reliability based design of SWATH ship structure. Proceedings of the Fifth International Conference on the Integrity of Offshore Structures; 1993 Jun 17-18; Glasgow, United Kingdom. Warley, UK: EMAS. p. 387-415.

Zanic V, Grubisic I, Trincas G. 1992. Multiattribute decision making system based on random generation of nondomi- nated solutions - application to fishing vessel design. Proceedings of the 3rd International Symposium on 'Practical Design of Ships and Mobile Units', PRADS'92; 1992 May 17-22; Newcastle upon Tyne, United Kingdom. Barking, UK: Elsevier.break; p. 623-630.

Zanic V, Kitarovic S, Prebeg P. 2010. Safety as objective in multi-criterial structural optimization. Proceedings of ASME/OMAE; 2010 Jun 6-11; Shanghai, China, New York: ASME.

Zitzler E., Laumanns M., Thiele L. 2001. SPEA2: improving the strength pareto evolutionary algorithm. Zurich (Switzerland): Eidgenössische Technische Hochschule Zürich (ETH). 